\title{
Risk Factors and Predictors of Severe Dengue in Saudi Population in Jeddah, Western Saudi Arabia: A Retrospective Study
}

\author{
Moustafa A. Hegazi, ${ }^{1,2 \star}$ Marwan A. Bakarman, ${ }^{3}$ Turki S. Alahmadi,,${ }^{1,4}$ Nadeem S. Butt, ${ }^{3}$ Ahmed M. Alqahtani, ${ }^{5}$ Badr S. Aljedaani, ${ }^{5}$ \\ and Abdulrahman $\mathrm{H}$. Almajnuni ${ }^{5}$ \\ ${ }^{1}$ Department of Pediatrics, Faculty of Medicine in Rabigh, King Abdulaziz University, Jeddah, Saudi Arabia; ${ }^{2}$ Department of Pediatrics, Mansoura \\ University Children's Hospital, Mansoura, Egypt; ${ }^{3}$ Department of Family and Community Medicine, Faculty of Medicine in Rabigh, King Abdulaziz \\ University, Jeddah, Saudi Arabia; ${ }^{4}$ Department of Pediatrics, King Abdulaziz University Hospital, Jeddah, Saudi Arabia; ${ }^{5}$ Faculty of Medicine in \\ Rabigh, King Abdulaziz University, Jeddah, Saudi Arabia
}

\begin{abstract}
This study was performed to determine the risk factors and predictors of severe dengue fever (SDF) in Saudi population in Jeddah, Western Saudi Arabia. This 7-year retrospective study included children and adults with confirmed dengue from 2010 to 2016. Demographic, clinical, laboratory, serological, and virologic data were collected. Comparative analyses were performed between pediatric and adult SDF cases defined according to the WHO 2009 dengue classification. During the study period, dengue was confirmed in 17,646 cases with predominant infection of adults $(6.5$ times that of children) and males (3.8 times that of females). May and June were associated with $43.9 \%$ of total dengue cases. All 56 pediatric and 187 adult SDF cases were hospitalized. At least one warning sign of severe illness was present in $92.2 \%$ of total SDF cases. Mortality rates were $8.9 \%$ and $10.7 \%$ of pediatric and adult SDF cases, respectively. Multiple logistic regression detected that the most significant risk factors and predictors of SDF in adults versus children were significantly more secondary dengue infection (adjusted odds ratio [AOR]:2.20, 95\% Cl: 1.09-4.44, $P=0.02$ ), significantly less clinical fluid accumulation (AOR: $0.17,95 \% \mathrm{Cl}: 0.07-0.44, P<0.001)$ and significantly less neutropenia (AOR: $0.41,95 \% \mathrm{Cl}$ : $0.17-0.97, P=0.04$ ). This was the first large multicenter study evaluating SDF in Saudi population and considering the WHO 2009 dengue classification, which showed predominant infection of adults and males with dengue, few SDF cases with low mortality and highlighted predictors of SDF in adults versus children. Consideration of warning signs for severe dengue may result in hospital admission, prompting closer monitoring, timely and proper interventions and reduced mortality in SDF cases.
\end{abstract}

\section{INTRODUCTION}

Dengue fever caused by four serotypes of dengue virus (DENV) is the most widespread mosquito-borne systemic acute viral infection affecting different populations. Dengue is endemic in all regions of the world except Europe. ${ }^{1,2}$

Although most DENV infections are asymptomatic or cause only mild systemic illness, small percentage of patients may develop serious complications. Severe complications such as dengue shock syndrome (DSS) and dengue hemorrhagic fever (DHF) occur relatively late in the course of the disease, giving a chance to pick up patients with high likelihood of progression to these complications. ${ }^{3}$

$\mathrm{WHO}$ dengue classification and management protocol was revised in 2009 to allow more effective recognition of cases of severe dengue fever (SDF) replacing dengue fever, DHF, and DSS by dengue without warning signs (D-WS), dengue with warning signs (D+WS), and SDF.,

Secondary dengue infection (SDI) is the most important risk factor of SDF which may be related to both host and viral factors based on the antibody-dependent enhancement mechanism suggesting that SDF coincides with SDI when antibodies produced in a primary infection augment binding of DENV-IgG antibodies to receptors on macrophages, increasing severity of infection. ${ }^{5}$

Risk factors identified for DHF, DSS, and SDF included younger age, SDI, persistent vomiting, abdominal pain, mucosal bleeding, lethargy, hepatomegaly, fluid accumulation, infection with DENV serotype 2, increased hematocrit (HCT)

*Address correspondence to Moustafa A. Hegazi, Department of Pediatrics, Faculty of Medicine in Rabigh, King Abdulaziz University, P.O. Box 80205, Jeddah 21589, Saudi Arabia. E-mails: mhegazi712003@ yahoo.co.uk or mahhassan@kau.edu.sa more than $22 \%$ from baseline, increased level of alanine aminotransferase (ALT) or aspartate aminotransferase (AST), thrombocytopenia, and coagulation abnormalities. ${ }^{6,7}$

In the presence of large population already infected with DENV, different DENV types, and introduction of recent strains or genetic alteration in existing strains, there is always a possibility of outbreaks of SDF. ${ }^{1}$ Dengue is now endemic in Saudi Arabia with favorable circumstances for dengue outbreaks already present in our locality, Jeddah, as at least three DENV serotypes are circulating and locally transmitted. ${ }^{8}$

For international health perspectives, dengue is not only an important viral infection for population in Jeddah, Western Saudi Arabia, but for many populations in other countries. Jeddah is a Haj access point with many pilgrims coming from especially Indonesia, Malaysia, and Thailand, which are high-burden dengue countries and from other dengue-endemic areas all over the world which allows persistent entry/exit of DENV different strains into/from Saudi Arabia. ${ }^{1,9}$

In a study from Jeddah, increasing the severity of illness from dengue fever to DHF or DSS was associated with a slight inverse correlation of the level of DENV IgM antibodies with patient age assuming that the younger the patient, the greater the possibility of a primary dengue infection. ${ }^{10}$

No published data or previous studies about risk factors of SDF have ever involved Saudi population considering the WHO 2009 dengue classification. Thus, this retrospective study was performed to determine demographic, clinical, laboratory, and virologic risk factors that were associated with SDF in adults versus children with confirmed dengue from 2010 to 2016 according to the WHO 2009 dengue classification, in Jeddah. 


\section{MATERIALS AND METHODS}

Study population. This retrospective multicenter study included children and adults with laboratory-confirmed diagnosis of dengue from the beginning of January 2010 to end of December 2016. Pediatric and adult dengue cases of different severity were defined according to revised $\mathrm{WHO}$ 2009 dengue classification guidelines. Cases presented more than 7 days from start of illness, cases with comorbid conditions or chronic diseases that may increase dengue severity, cases with unconfirmed diagnosis of dengue or incomplete data, and non-Saudi patients were excluded from this study.

Data collection. A well-designed data collection form was used to gather data from patients' records at the Jeddah dengue control center of Saudi Ministry of Health, which receives patients' data as well as patients' serum samples to perform dengue serology and confirmatory tests, from governmental and private health-care centers in Jeddah. This form had almost all possible relevant risk factors of dengue, including complete demographic, clinical, laboratory, serological, and virologic data. There is a continuous communication between Jeddah dengue control center and health-care centers to inform them about results of tests and confirmed dengue cases and to receive updated data about progress and final outcome of dengue cases from health-care centers. To guarantee accurate data collection, each patient's record was double-checked to ensure confirmed diagnosis of dengue, subsequently categorized as SDF or non-severe dengue fever (Non-SDF) according to the WHO 2009 dengue classification.

As early identification of selection criteria of SDF cases on initial presentation is extremely important in further management, this study used the demographic, clinical, and laboratory data of patients on hospital admission in addition to their final outcome of survival or death.

Laboratory data included recording red blood cell counts (RBCs), white blood cell counts (WBCs) with differential leukocytic count, platelet count, hemoglobin (HB), hematocrit (HCT), liver functions tests (total serum bilirubin [TSB], direct serum bilirubin [DSB], serum albumin and liver enzymes [AST and $A L T]$ ), and serum creatinine. Serology included DENV-IgM and DENV-IgG antibodies. Dengue confirmatory data by DENV polymerase chain reaction (DENV-PCR) and nonstructural protein antigen 1 (NS1) were also included. Dengue virus serotypes were included whenever available.

Definitions. Pediatric cases were considered if age was up to 18 years, whereas adults had age $>18$ years.

According to the $\mathrm{WHO} 2009$ dengue classification, dengue cases were divided into two groups: Non-SDF and SDF. NonSDF included two subgroups: D-WS and D+WS cases. Patients with D-WS had fever and two of the following criteria: anorexia and nausea, aches and pains, rash, leukopenia, positive tourniquet test, and laboratory-confirmed dengue, with ability to tolerate adequate volumes of oral fluid replacement and to pass urine at least once every 6 hours. Dengue cases with warning signs (D+WS) were defined as patients with any of the following signs: abdominal pain/tenderness, persistent vomiting, lethargy, liver enlargement $>2 \mathrm{~cm}$, mucosal bleeding, clinical fluid accumulation, or increasing HCT with decreasing platelets. The SDF group included patients with any of the following criteria: severe bleeding $(\mathrm{SDF}+\mathrm{SB})$, severe plasma leakage with shock
(SDF+DSS), fluid accumulation with respiratory distress (SDF+FA), or severe organ involvement $(\mathrm{SDF}+\mathrm{SOI}){ }^{1}$

Laboratory-confirmed dengue cases included those with febrile illness suggestive of dengue and positive DENV-IgM and DENV-IgG confirmed by positive dengue NS1 antigen capture ELISA (Panbio Dengue Early ELISA, Alere, Brisbane, Australia) test and DENV-PCR.

Serologic diagnosis of all included dengue cases was based on the presence of DENV-IgM and DENV-IgG in acutephase serum from day 4 to 7 of the onset of dengue infection by ELISA tests (Panbio Dengue IgM Capture ELISA and Panbio Dengue IgG Capture ELISA) manufactured by Panbio (Alere). Primary and SDI cases were based on negative or positive DENV-IgG capture ELISA, respectively, confirmed by dengue NS1 antigen and DENV-PCR. Panbio dengue IgG assay does not detect low-threshold DENV-lgG titer from primary dengue affecting large populations in dengueendemic regions but is designed to reveal high DENV-IgG titer above this threshold. ${ }^{11,12}$ Moreover, it was found that dengue capture IgG ELIZA test is a preferred method after day 4 of illness to differentiate primary from SDI dengue during acute phase of dengue infection, ${ }^{13}$ and serologic tests were applied for the included patients from day 4 to 7 from the onset of symptoms in this study.

Data analysis. The IBM SPSS version 25.0 (IBM corporation, Armonk, NY) was used to analyze data. Data were scrutinized and double-checked before and after entry into SPSS program. Categorical variables were presented as percentages, whereas continuous variables were presented as means and standard deviations (SDs). Chi-squared test was used to compare qualitative data between pediatric and adult SDF cases, whereas independent samples $t$-test was used to compare between means. Univariate logistic regression with calculation of unadjusted odds ratios (ORs) and 95\% Cls were performed to determine association between each variable and outcome variable (SDF). Multivariate logistic regression was performed to calculate adjusted ORs (AORs) and determine the most significant risk factors or predictors of SDF in adults versus children. Significance was considered at $P$-value $<0.05$.

Ethical approval. This study was approved by the research ethics committee of Faculty of Medicine in Rabigh and Medical Research and Studies Department of Directorate of Health Affairs-Jeddah of Saudi Ministry of Health.

\section{RESULTS}

During the study period, there were 17,646 confirmed dengue cases in both adults and children. Dengue was confirmed in all Non-SDF and SDF cases by positive NS1 antigen. Additional confirmation by positive DENV-PCR was also performed in 15,244 (87.6\%) of Non-SDF and 223 (91.8\%) of SDF cases.

Adult cases of dengue represented about 6.5 times that of pediatric cases $(15,280$ in adults versus 2,366 in children). Dengue cases were predominant in male gender; irrespective of adults or children, male cases were about 3.8 times that of female cases (13,983 males versus 3,663 females). There were 1,628 male cases $(68.8 \%)$ in children and 12,355 cases in adults (80.9\%) with significantly higher adult males (OR: 1.91, 95\% Cl: 1.74-2.11, $P<0.0001)$. Figure 1 shows the study flowchart with total number of confirmed dengue cases and 


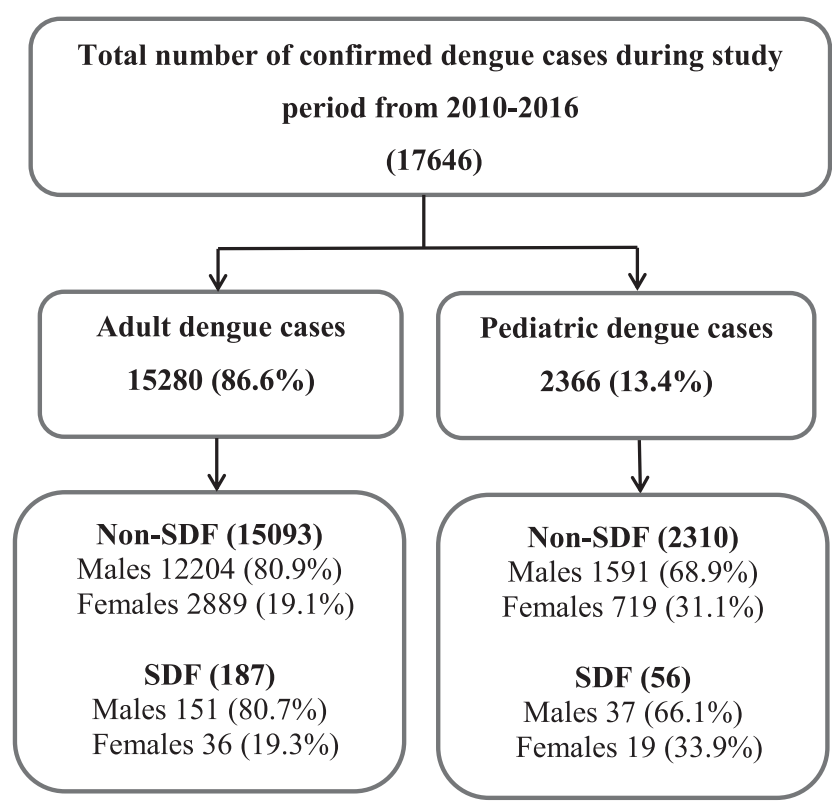

FIGURE 1. Study flowchart with total number of confirmed dengue cases and their distribution according to the WHO 2009 dengue infection classification during the 7-year study period from 2010 to 2016 in adults and children.

their distribution according to the WHO 2009 dengue infection classification during the study period in adults and children.

According to the WHO 2009 dengue classification, 17,403 Non-SDF cases including D-WS and D+WS cases versus 243 SDF cases were identified, including 56 pediatric and 187 adult SDF cases representing $2.4 \%$ and $1.2 \%$ of pediatric and adult dengue cases, respectively. The mean and SD of age of Non-SDF and SDF cases were $10.9 \pm 5.4$ and $12.1 \pm 4.5$ years, respectively, in children $(P=0.09)$, whereas mean and SD of age were significantly higher in SDF adult cases (38.7 \pm 13.9 years) compared with Non-SDF adult cases $(35.9 \pm 9.8$ years, $P=0.0001$ ).

The highest urban areas with dengue infection were from center with 5,024 cases $(28.47 \%)$ and south Jeddah with 4,867 cases $(27.58 \%)$. South Jeddah had significantly more SDF cases than north Jeddah $(P=0.001$, Table 1$)$.

Regarding monthly distribution of dengue cases, May and June had the highest rates of dengue infection as they were associated with $43.9 \%$ of total dengue cases. Figure 2 shows monthly distribution of total dengue cases during the study period.

Comparison between SDF and Non-SDF cases (Table 1) revealed that non-warning signs such as headache and maculopapular skin rash were significantly more in Non-SDF cases than in SDF cases $(P=0.02$ and 0.0001 , respectively). Cases with SDF were significantly more associated with residence in south Jeddah $(P=0.001)$, SDI $(P<0.0001)$, warning signs of the WHO 2009 classification apart from persistent vomiting, significantly higher levels of RBCs, HB, HCT, WBCs, hepatic enzymes, and serum creatinine and significantly lower platelet count and serum albumin compared to Non-SDF cases.

Pediatric cases had significantly more risk to develop SDF (OR: $1.96,95 \% \mathrm{Cl}: 1.45-2.65, P<0.0001)$. The mean and SD of age of SDF cases were $12.1 \pm 4.5$ years with a range from 1 to 17 years in children and $38.7 \pm 13.9$ years with a range from 20 to 76 years in adults. Male gender was predominant in both children and adults and comprised $80.7 \%$ of adults which was significantly higher than $66.1 \%$ in children (OR: $2.2,95 \% \mathrm{Cl}: 1.1-4.2, P=0.02)$. South Jeddah was the urban area with the highest number of SDF cases with 24 cases $(42.9 \%)$ in children and 66 cases in adults (35.3\%) but without significant differences from other areas.

Adult SDF cases had significantly more SDI than pediatric SDF cases (OR: $2.46,95 \% \mathrm{Cl}: 1.33-4.57, P=0.004)$, and significantly more adults than children were admitted to intensive care units (ICU) (OR: 2.23, 95\% Cl: 1.02-4.86, $P=0.04)$ Table 2 .

Most SDF cases survived with recorded death of five cases (8.9\%) in children and 20 cases $(10.7 \%)$ in adults without significant difference between children and adults (Table 2). The overall case fatality rate (CFR) of SDF cases in relation to total dengue cases was only $0.14 \%$. In children, the five SDF deaths included four males and one female with a median age of 9 years. Only one male with SDF had SDI. Two male pediatric SDF dead cases had SDF+SOI involving the brain with frequent seizures and coma and mild thrombocytopenia. The other three dead pediatric SDF cases had SDF+DSS, SDF+SB, severe thrombocytopenia, SDF+FA, and multiple SOI involving brain, lung, and liver with markedly elevated hepatic enzymes. On the other hand, all dead adult SDF cases were males with a median age of 51.5 years and nine cases were older than 56 years. Twelve dead SDF adult cases (60\%) had SDI. In dead adults, 17 had SOI involving brain, liver, lung, and kidney; five had SDF+DSS; 10 had SDF+SB with severe thrombocytopenia; and seven had SDF+FA.

Regarding clinical manifestations of SDF cases, at least one warning sign was present in $92.2 \%$ of total SDF cases. Persistent vomiting, abdominal pain, and lethargy were the most common warning signs present in $>50 \%$ of SDF cases. Persistent vomiting and respiratory distress were more significantly recorded in children than in adults $(P=0.003$ and 0.002 , respectively). Nonsignificant differences were detected in all forms of SDF (SB, DSS, and SOI) between children and adults. All forms of clinical fluid accumulation were more significantly found in children than in adults $(P<0.0001)$. The most common forms of bleeding were mucosal and cutaneous bleeding $(28.6 \%$ of pediatric SDF cases and $32.6 \%$ of adult SDF cases) followed by epistaxis (28.6\% and $29.9 \%$ in pediatric and adult SDF cases, respectively). Table 2 presents the clinical data and manifestations of SDF cases.

The mean values of RBCs, HB, HCT, and WBCs were significantly higher in adults than in children. Adult SDF cases had significantly less neutropenia and mild neutropenia and significantly more moderate thrombocytopenia than pediatric SDF cases (Table 3).

The mean values of ALT, AST, TSB, DSB, and serum albumin were not significantly different between pediatric and adult SDF cases $(P>0.05)$. Serum creatinine was significantly raised in adult SDF cases than in pediatric SDF cases $(P<0.0001)$ (Table 3).

Multivariate regression analysis detected that the most significant independent risk factors or predictors of SDF in adults versus children were significantly more SDI (AOR: 2.20, 95\% Cl: 1.09-4.44, $P=0.02$ ), significantly less clinical fluid accumulation (AOR: 0.17, 95\% Cl: $0.07-0.44, P<0.001$ ), and significantly less neutropenia (AOR: $0.41,95 \% \mathrm{Cl}: 0.17-0.97$, $P=0.04$ ) (Table 4). The predictability for SDF of this logistic 
TABLE 1

Comparison between severe and non-severe dengue cases

\begin{tabular}{|c|c|c|c|c|}
\hline Variables & Non-severe dengue $N=17,403$ & Severe dengue $N=243$ & Odds ratio $(95 \% \mathrm{Cl})$ & $P$-values \\
\hline \multicolumn{5}{|l|}{ Gender } \\
\hline Male & 13,795 (79.3) & $188(77.4)$ & $0.77(0.567-1.04)$ & $0.08^{\star}$ \\
\hline Female & $3,608(20.7)$ & $55(22.6)$ & - & - \\
\hline \multicolumn{5}{|l|}{ Residence } \\
\hline Urban north $(N, \%) \dagger$ & $2,852(16.39)$ & $26(10.70)$ & NA & NA \\
\hline Urban south $(N, \%)$ & $4,777(27.45)$ & $90(37.04)$ & $2.07(1.33-3.21)$ & $0.001^{*}$ \\
\hline Urban east $(N, \%)$ & $1,709(9.82)$ & $22(9.05)$ & $1.41(0.79-2.49)$ & $0.24^{*}$ \\
\hline Urban west $(N, \%)$ & $3,105(17.84)$ & $41(16.87)$ & $1.45(0.88-2.37)$ & $0.14^{\star}$ \\
\hline Urban center $(N, \%)$ & $4,960(28.50)$ & 64 (26.34) & $1.42(0.89-2.24)$ & $0.14^{*}$ \\
\hline Secondary dengue infection (Yes) & $4,260(24.5)$ & $128(52.7)$ & $3.43(2.66-4.43)$ & $<0.0001^{*}$ \\
\hline Hospital admission & $10,501(60.3)$ & $243(100)$ & NA & $<0.0001^{*}$ \\
\hline \multicolumn{5}{|l|}{ Non-warning manifestations } \\
\hline Headache $(N, \%)$ & $14,149(81.3)$ & $183(75.3)$ & $0.70(0.52-0.94)$ & $0.02^{*}$ \\
\hline Musculoskeletal pain $(N, \%)$ & $11,747(67.5)$ & $157(64.6)$ & $0.88(0.67-1.15)$ & $0.34^{*}$ \\
\hline Maculopapular skin rash $(N, \%)$ & $5,691(32.7)$ & $50(20.6)$ & $0.53(0.39-0.73)$ & $0.0001^{*}$ \\
\hline \multicolumn{5}{|l|}{$\begin{array}{l}\text { Warning signs of the WHO } 2009 \text { dengue } \\
\text { classification }\end{array}$} \\
\hline Abdominal pain or tenderness $(N, \%)$ & $6,074(34.9)$ & $139(57.2)$ & $2.49(1.93-3.22)$ & $<0.0001^{*}$ \\
\hline Persistent vomiting (N, \%) & $10,407(59.8)$ & $154(63.4)$ & $0.16(0.89-1.51)$ & $0.26^{\star}$ \\
\hline Mucosal bleeding $(N, \%)$ & $226(1.3)$ & $13(5.3)$ & $4.30(2.42-7.63)$ & $<0.0001^{*}$ \\
\hline Hepatomegaly $(N, \%)$ & $592(3.4)$ & $62(25.5)$ & $9.73(7.21-13.13)$ & $<0.0001^{*}$ \\
\hline Lethargy $(N, \%)$ & $10,163(58.4)$ & $191(78.6)$ & $2.62(1.93-3.56)$ & $<0.0001^{*}$ \\
\hline Clinical fluid accumulation $(N, \%)$ & $418(2.4)$ & $32(13.2)$ & $6.16(4.19-9.05)$ & $<0.0001^{*}$ \\
\hline Increased HCT with thrombocytopenia ( $N, \%)$ & $278(1.6)$ & 34 (14) & $10.02(6.84-14.67)$ & $<0.0001^{*}$ \\
\hline \multicolumn{5}{|l|}{ Hematological and biochemical laboratory data } \\
\hline Red blood cells (mean $\pm \mathrm{SD})\left(\times 10^{3} / \mathrm{mm}^{3}\right)$ & $4.23 \pm 0.63$ & $4.79 \pm 0.85$ & NA & $<0.0001 \ddagger$ \\
\hline Hemoglobin (mean $\pm \mathrm{SD})(\mathrm{g} / \mathrm{dL})$ & $12.78 \pm 2.45$ & $13.35 \pm 2.86$ & NA & $0.0003 \ddagger$ \\
\hline Hematocrit \% (mean \pm SD) & $38.41 \pm 7.62$ & $39.58 \pm 8.25$ & NA & $0.02 \ddagger$ \\
\hline White blood cells (mean \pm SD) $\left(\times 10^{3} / \mathrm{mm}^{3}\right)$ & $3.14 \pm 1.01$ & $3.43 \pm 1.11$ & NA & $<0.0001 \ddagger$ \\
\hline Platelet count (mean $\pm \mathrm{SD})\left(\times 10^{3} / \mathrm{mm}^{3}\right)$ & $133.10 \pm 42.68$ & $79.56 \pm 25.13$ & NA & $<0.0001 \ddagger$ \\
\hline Alanine aminotransferase (mean $\pm \mathrm{SD})(\mathrm{IU} / \mathrm{L})$ & $63.35 \pm 15.16$ & $133.32 \pm 31.39$ & NA & $<0.0001 \ddagger$ \\
\hline Aspartate aminotransferase (mean \pm SD) (IU/L) & $96.15 \pm 31.87$ & $244.25 \pm 66.64$ & NA & $<0.0001 \ddagger$ \\
\hline Total serum bilirubin (mean $\pm \mathrm{SD}$ ) (mg/dL) & $0.83 \pm 0.18$ & $2.29 \pm 0.59$ & NA & $<0.0001 \ddagger$ \\
\hline Direct serum bilirubin (mean $\pm \mathrm{SD})(\mathrm{mg} / \mathrm{dL})$ & $0.23 \pm 0.02$ & $0.44 \pm 0.14$ & NA & $<0.0001 \ddagger$ \\
\hline Serum albumin (mean $\pm \mathrm{SD})(\mathrm{g} / \mathrm{dL})$ & $3.63 \pm 0.55$ & $3.16 \pm 1.09$ & NA & $<0.0001 \ddagger$ \\
\hline Serum creatinine $($ mean $\pm \mathrm{SD})(\mathrm{mg} / \mathrm{dL})$ & $0.66 \pm 0.13$ & $1.03 \pm 0.37$ & NA & $<0.0001 \ddagger$ \\
\hline
\end{tabular}

* Chi-squared test.

† Base or reference category.

$\ddagger$ Independent $t$-test.

model reached $94.1 \%$ in adults with overall accuracy of $77.4 \%$ (Figure 3).

The DENV serotyping data were available for 57 (23.5\%) of SDF cases; including 18 pediatric SDF cases and 39 adult SDF cases. The detected DENV serotypes included DENV-1, DENV-2, and DENV-3 in 19, 24, and 14 of total SDF.

\section{DISCUSSION}

In this study, dengue infection in Saudi population in Jeddah was associated with definite characteristics with overall predominant infection of more adults than children and more males than females and living in center and south Jeddah. This runs in concordance with other studies as there has been a notable increase in age of dengue cases to involve older children and adults and more males than females. ${ }^{14,15}$ Predominant infection of more adults than children and more males than females might be related to more exposure of adults and males to mosquitos' bites during their more frequent outdoor activities. The associations of dengue cases with males, adolescents, and young adults with most cases occurring in May and June and residence in south Jeddah was also reported in other studies in Jeddah. ${ }^{9,16,17}$ The association of dengue infection with certain season and residential areas could be related to favorable conditions for growth and breeding of the mosquito vector.

Considering the revised $\mathrm{WHO} 2009$ dengue classification, the comparison between SDF and Non-SDF cases (Table 1) revealed that residence in south Jeddah, SDI, warning signs of the WHO 2009 dengue classification apart from persistent vomiting, higher levels of RBCs, HB, HCT, WBCs, hepatic enzymes and serum creatinine, and lower levels of platelet count and serum albumin were more significantly associated with SDF cases compared with Non-SDF cases which had been recorded in previous worldwide and Saudi studies. ${ }^{5-7,10}$ Moreover, the values of simple easily recognizable alarming or warning signs in the WHO 2009 dengue classification and routine laboratory tests on initial presentation or admission seemed to be greatly helpful in accurate assessment and discrimination of severe cases, hospital admission decision for all SDF cases (Table 2) and proper management of dengue patients expected to develop SDF.

In this study, further in depth analysis focusing mainly on SDF cases comparing between pediatric and adults SDF cases was undertaken to identify age-related risk factors of SDF, which may represent an important approach in elucidating pathogenesis of SDF which was not explored in many 


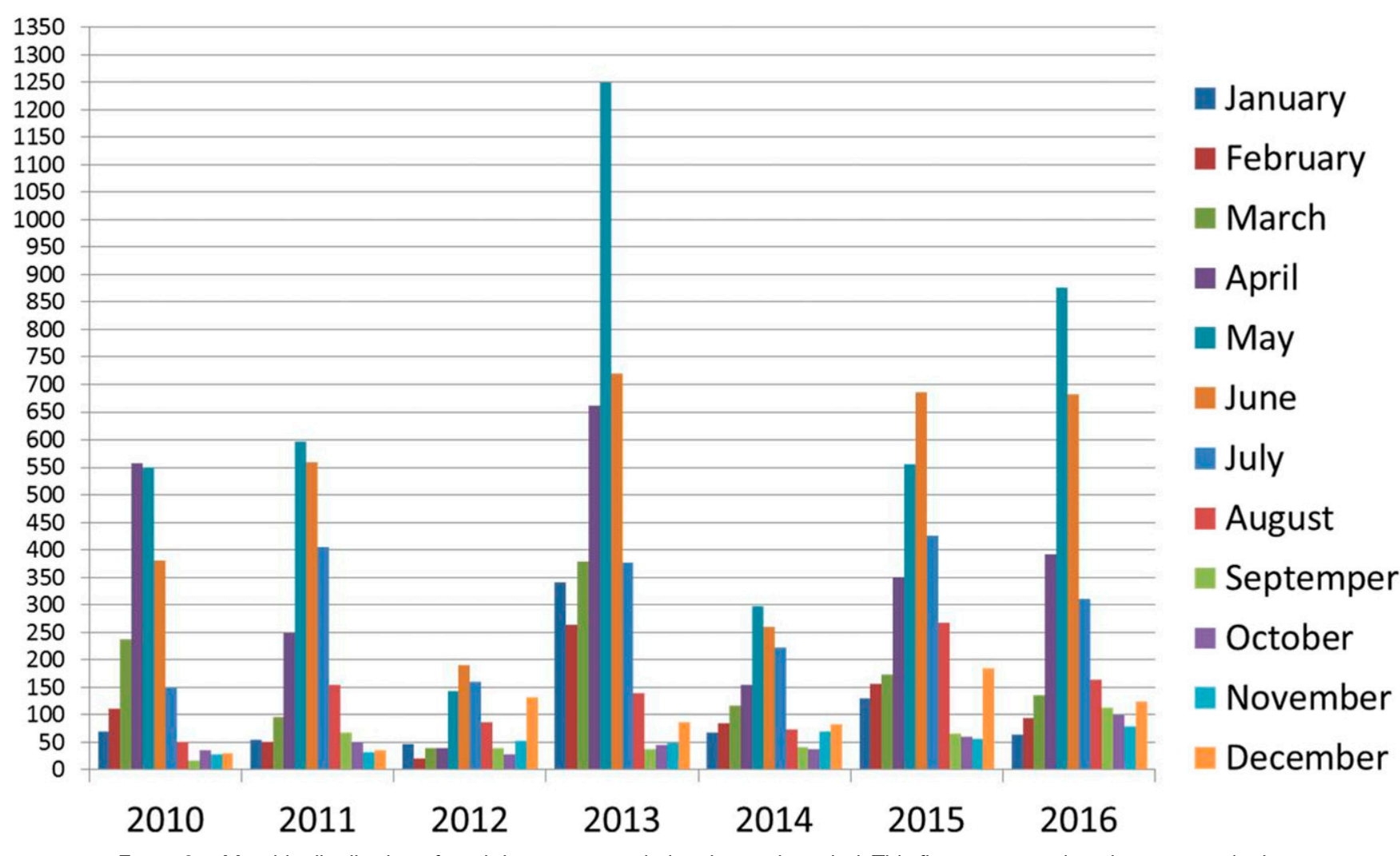

FIGURE 2. Monthly distribution of total dengue cases during the study period. This figure appears in color at www.ajtmh.org.

published previous studies, especially Saudi studies about dengue fever.

Pediatric cases had significantly more risk to develop SDF. Similarly, other studies showed more severity of dengue infection, especially DSS in children. ${ }^{6,7}$ However, in this study, death was only recorded in $8.9 \%$ and $10.7 \%$ of pediatric and adult SDF cases, respectively, with an overall CFR of 0.14 comparable with CFR of SDF forms ranging from $0.2 \%$ to $1.1 \%$ recorded in other studies. ${ }^{18-20}$ This low CFR could be related to rapid diagnosis of cases by high index of suspicion because of known locality-dengue risk, hospitalization and proper management protocol of SDF cases at tertiary healthcare centers with adequate resources. Moreover, the presence of at least one warning sign in most SDF cases most probably resulted in hospital admission decision in anticipation of more severe illness with close monitoring, ICU admission, and timely proper interventions for such severe cases. This can point to the usefulness of the WHO 2009 dengue classification in early identification, categorization, and management of dengue cases at risk of progression to SDF. The WHO 2009 dengue classification demonstrated the ability to distinguish risk factors of SDF with excellent sensitivity and specificity for timely recognition of SDF cases in need for accurate early decisions, extensive care, and proper management with higher benefit and lower cost than the 1997 WHO scheme. ${ }^{2,4}$ Furthermore, the required extreme rigorous care for SDF cases could have been optimally provided because of the established dengue surveillance and management system in Saudi Arabia, especially in the western region that could effectively deal with large numbers of annual dengue cases during the period from 2010 to 2016 which even exceed the numbers previously reported in the three major epidemics in Saudi Arabia: DENV-2 epidemic in 1994, DENV-1 epidemic in 2006, and the last DENV-3 epidemic in $2008 .^{1}$

Although children had more risk than adults to develop SDF, the degree of severity of illness seemed to be slightly more in adults who were more significantly admitted to ICU with slightly more death percentage among adult than pediatric SDF cases. In a Brazilian study, adults also had a higher frequency of SDF and hospitalization relative to children. ${ }^{21}$ These findings may indicate a trend toward an increasing severity of illness with advancing age that can be mostly attributed to more possibility to get SDI as age increases, which is the most important risk factor for $\mathrm{SDF}^{2,5,10,22}$ and possibility of adults, especially the elderly to have more subtle unrecognized organ impairments than children. Also, it was demonstrated in this study that adult SDF cases had more multiple SOI than pediatric SDF cases, but it did not reach statistical significance $(P=0.09$, Table 2$)$. Moreover, in the current study, a possible contributory adverse effect of aging on kidney functions was recognized by finding significantly higher serum creatinine in adults than pediatric SDF cases $(P<0.001$, Table 3). Similarly, serum creatinine was significantly higher on admission among fatal adult SDF cases. ${ }^{23}$

Both factors of SDI and older age were evident in this study where SDI was a significant predictor of SDF in adults compared with children in regression analysis and nine $(45 \%)$ of dead SDF adults were older than 56 years. Similarly, it was found that the best predictor of death in SDF was age greater than 55 years ${ }^{24}$ and likelihood of death increased by 2.5 -folds per each 10-year increase in age. ${ }^{25}$

The main hematologic parameter which was significantly higher in adult SDF than pediatric SDF was mean WBCs $(P<$ 0.0001 , Table 3 ), and less neutropenia was a significant 
TABLE 2

Comparison of clinical data and manifestations between pediatric and adult severe dengue fever (SDF) cases

\begin{tabular}{|c|c|c|c|c|}
\hline Variables & Pediatric SDF, $N=56(N, \%)$ & Adult SDF, $N=187(N, \%)$ & Odds ratio $(95 \% \mathrm{Cl})$ & $P$-values \\
\hline Secondary dengue infection & $20(35.7)$ & $108(57.8)$ & $2.46(1.33-4.57)$ & 0.004 \\
\hline \multicolumn{5}{|l|}{ Management/place of admission } \\
\hline Ward admission & 47 (83.9) & $131(70.1)$ & - & - \\
\hline Intensive care unit admission & $9(16.1)$ & $56(29.9)$ & $2.23(1.02-4.86)$ & 0.04 \\
\hline \multicolumn{5}{|l|}{ Outcome } \\
\hline Survived & $51(91.1)$ & $167(89.3)$ & - & - \\
\hline Died & $5(8.9)$ & $20(10.7)$ & $1.22(0.44-3.42)$ & 0.70 \\
\hline \multicolumn{5}{|l|}{ Non-warning manifestations } \\
\hline Headache & 39 (69.6) & $144(77)$ & $1.46(0.75-2.83)$ & 0.26 \\
\hline Musculoskeletal pain & 30 (53.6) & $127(67.9)$ & $1.83(0.99-3.37)$ & 0.05 \\
\hline Maculopapular skin rash & $13(23.2)$ & $37(19.8)$ & $0.82(0.39-1.67)$ & 0.58 \\
\hline \multicolumn{5}{|l|}{$\begin{array}{l}\text { Warning signs of the WHO } 2009 \text { dengue } \\
\text { classification }\end{array}$} \\
\hline Abdominal pain or tenderness & $31(55.4)$ & $108(57.8)$ & $1.10(0.60-2.01)$ & 0.75 \\
\hline Persistent vomiting & $45(80.4)$ & $109(58.3)$ & $0.34(0.17-0.70)$ & 0.003 \\
\hline Mucosal bleeding & $5(8.9)$ & $8(16)$ & $0.95(0.33-2.73)$ & 0.93 \\
\hline Hepatomegaly & $13(23.2)$ & 49 (26.2) & $1.17(0.58-2.37)$ & 0.65 \\
\hline Lethargy & 47 (83.9) & $144(77)$ & $0.64(0.29-1.41)$ & 0.27 \\
\hline Clinical fluid accumulation (all forms) & $17(30.4)$ & $15(8.0)$ & $0.19(0.09-0.43)$ & $<0.0001$ \\
\hline Ascites & $2(3.6)$ & $2(1.1)$ & $0.22(0.03-1.63)$ & 0.14 \\
\hline Pleural effusion & $3(5.4)$ & $4(2.12)$ & $0.29(0.06-1.38)$ & 0.12 \\
\hline Both ascites and pleural effusion & $12(21.4)$ & $9(4.8)$ & $0.17(0.07-0.42)$ & 0.0002 \\
\hline No plasma leakage* & $35(62.5)$ & $158(84.5)$ & 3.27 (1.67-6.39) & 0.0005 \\
\hline $\begin{array}{l}\text { Increased hematocrit value with } \\
\text { thrombocytopenia }\end{array}$ & $6(10.7)$ & $28(15.7)$ & $1.47(0.57-3.75)$ & 0.42 \\
\hline \multicolumn{5}{|l|}{ Other clinical manifestations } \\
\hline Seizures & $6(10.7)$ & $33(17.6)$ & $1.79(0.70-4.51)$ & 0.22 \\
\hline Respiratory distress & $10(17.9)$ & $8(4.28)$ & $0.21(0.08-0.55)$ & 0.002 \\
\hline Bleeding (all forms) & 47 (83.93) & $157(84.0)$ & $1.0(0.44-2.26)$ & 0.99 \\
\hline Hematemesis/melena & $5(8.9)$ & $16(8.6)$ & $0.96(0.28-3.35)$ & 0.95 \\
\hline Epistaxis & $16(28.6)$ & $56(29.9)$ & $1.05(0.42-2.66)$ & 0.92 \\
\hline Purpura/ecchymosis & $5(8.9)$ & $8(4.3)$ & $0.48(0.13-1.84)$ & 0.28 \\
\hline Gum bleeding & $5(8.9)$ & $16(8.6)$ & $0.96(0.28-3.35)$ & 0.95 \\
\hline Cutaneous and mucosal bleeding & $16(28.6)$ & 61 (32.6) & $1.14(0.45-2.89)$ & 0.78 \\
\hline Severe bleeding $\dagger$ & $9(16.1)$ & $17(9.1)$ & $0.52(0.22-1.25)$ & 0.14 \\
\hline No bleeding* & $9(16.1)$ & $30(16)$ & $0.99(0.44-2.25)$ & 0.99 \\
\hline Shock (dengue shock syndrome) & $4(7.1)$ & $14(7.5)$ & $1.05(0.33-3.33)$ & 0.93 \\
\hline Organ failure (all forms) & $17(30.4)$ & $70(37.4)$ & $1.37(0.72-2.61)$ & 0.33 \\
\hline Brain & $2(3.6)$ & $9(4.8)$ & $1.50(0.31-7.24)$ & 0.61 \\
\hline Liver & $12(21.4)$ & $24(12.8)$ & $0.67(0.31-1.46)$ & 0.31 \\
\hline Brain and liver & $1(1.8)$ & $15(8.0)$ & $5.0(0.64-39.09)$ & 0.13 \\
\hline Multiple & $2(3.6)$ & $22(11.8)$ & $3.67(0.83-16.31)$ & 0.09 \\
\hline No organ failure* & 39 (69.6) & 117 (62.6) & $0.73(0.38-1.38)$ & 0.33 \\
\hline
\end{tabular}

† Bleeding associated with systolic hypotension, hemoglobin $<8 \mathrm{~g} / \mathrm{dL}$, or bleeding that required blood transfusion.

predictor of SDF in adults by regression analysis. This finding was also identified in other studies as fatal adult SDF cases had higher WBCs on admission compared with nonfatal matched controls. ${ }^{23,26}$

Another identified significant predictor of SDF in adults compared with children in multivariate regression analysis was significantly less clinical fluid accumulation. Comparably, the same was recorded as plasma leakage may be marked, especially in children, occasionally resulting in fatal DSS. ${ }^{27}$ Also, clinical fluid accumulation with ascites and pleural effusion were predictors of pediatric SDF. ${ }^{28,29}$ Trung et al., ${ }^{30}$ also showed that children had more frequent and more severe plasma leakage and shock than adults, whereas organ involvement, SDI-associated severe thrombocytopenia, and bleeding manifestations were more common in adults.

Several points of strength and unique characteristics were evident in this study. It is the first multicenter comprehensive study which thoroughly characterized confirmed SDF (by NS1/PCR) in Saudi adults versus children applying the revised
WHO 2009 dengue guidelines on large number of dengue cases during 7 years and highlighted some age-related differences in dengue severity with investigation of almost all risk factors that could be associated with increasing dengue severity. The exact definition and classification of confirmed dengue cases, study design, proper data collection, and in depth systematic regression analyses with calculations of AOR allowed for efficient measurement of the role of each factor associated with SDF. The risk factors of SDF identified in this study should be useful in future anticipatory guidance and preventive measures especially for SDF in our locality as well as in other communities with similar conditions. Finally, the detected age-related differences in dengue severity may help in elucidating pathogenesis of SDF and developing appropriate guidelines for different age-groups at the risk of SDF.

Limitations of the study. Some limitations of this study included the retrospective nature of the study with inability to have serial measurements of underlying host immunological reactions and DENV-related risk factors for SDF as level of 
TABLE 3

Comparison of hematologic and biochemical laboratory data between pediatric and adult severe dengue fever (SDF) cases

\begin{tabular}{|c|c|c|c|c|}
\hline Variables & Pediatric SDF, $N=56$ & Adult SDF, $N=187$ & Odds ratio $(95 \% \mathrm{Cl})$ & $P$-values \\
\hline Hemoglobin (mean \pm SD) $\mathrm{g} / \mathrm{dL}$ & $12.71 \pm 2.92$ & $14.13 \pm 3.40$ & NA & $0.005^{\star} \dagger$ \\
\hline Red blood cells (mean \pm SD) $\times 10^{3} / \mathrm{mm}^{3}$ & $4.64 \pm 0.83$ & $4.95 \pm 0.88$ & NA & $0.02^{*} \dagger$ \\
\hline Hematocrit (mean $\pm S D$ ) \% & $38.27 \pm 7.40$ & $40.69 \pm 8.13$ & NA & $0.04^{*} \dagger$ \\
\hline White blood cells (mean $\pm \mathrm{SD}$ ) $\times 10^{3} / \mathrm{mm}^{3}$ & $2.92 \pm 1.31$ & $3.90 \pm 1.39$ & NA & $<0.0001 \dagger$ \\
\hline \multicolumn{5}{|l|}{ Neutropenia } \\
\hline No $(N, \%)$ & $25(44.6)$ & $136(72.7)$ & $3.31(1.78-6.13)$ & $0.0001 \ddagger$ \\
\hline $\operatorname{Mild}(N, \%)$ & $26(46.4)$ & $29(15.5)$ & $0.21(0.11-0.41)$ & $<0.0001 \ddagger$ \\
\hline Moderate $(N, \%)$ & $4(7.1)$ & $19(10.2)$ & $1.47(0.48-4.52)$ & $0.50 \ddagger$ \\
\hline Severe $(N, \%)$ & $1(1.9)$ & $3(1.6)$ & $0.89(0.09-8.79)$ & $0.93 \ddagger$ \\
\hline Platelet count (mean $\pm \mathrm{SD}) \times 10^{3} / \mathrm{mm}^{3}$ & $81.25 \pm 26.81$ & $78.12 \pm 23.44$ & NA & $0.39 \dagger$ \\
\hline \multicolumn{5}{|l|}{ Thrombocytopenia } \\
\hline $\mathrm{NO}(N, \%)$ & $10(17.9)$ & $10(5.3)$ & $0.26(0.10-0.66)$ & $0.005 \ddagger$ \\
\hline Mild $(N, \%)$ & $9(16.1)$ & $43(23)$ & $1.56(0.71-3.44)$ & $0.27 \ddagger$ \\
\hline Moderate $(N, \%)$ & $5(8.9)$ & $50(26.7)$ & $3.72(1.41-9.86)$ & $0.008 \ddagger$ \\
\hline Severe $(N, \%)$ & $32(57.1)$ & $84(45)$ & $0.61(0.33-1.12)$ & $0.11 \ddagger$ \\
\hline Alanine aminotransferase (mean $\pm \mathrm{SD}$ ) IU/L & $131.91 \pm 29.12$ & $134.74 \pm 33.65$ & NA & $0.57 \dagger$ \\
\hline Aspartate aminotransferase (mean \pm SD) IU/L & $245.75 \pm 81.10$ & $248.31 \pm 52.15$ & NA & $0.78 \dagger$ \\
\hline Total serum bilirubin (mean $\pm \mathrm{SD}$ ) $\mathrm{mg} / \mathrm{dL}$ & $2.25 \pm 0.63$ & $2.34 \pm 0.56$ & NA & $0.31 \dagger$ \\
\hline Direct serum bilirubin (mean $\pm \mathrm{SD}$ ) $\mathrm{mg} / \mathrm{dL}$ & $0.43 \pm 0.14$ & $0.45 \pm 0.09$ & NA & $0.21 \dagger$ \\
\hline Serum albumin (mean $\pm \mathrm{SD}$ ) $\mathrm{g} / \mathrm{dL}$ & $3.11 \pm 1.21$ & $3.20 \pm 0.98$ & NA & $0.57 \dagger$ \\
\hline Serum creatinine (mean \pm SD) $\mathrm{mg} / \mathrm{dL}$ & $0.63 \pm 0.28$ & $1.42 \pm 0.46$ & NA & $<0.0001^{*} \dagger$ \\
\hline
\end{tabular}

viremia, DENV virulence, and determination of DENV serotype for only $23.5 \%$ of SDF patients which hindered assessment of roles of these DENV-related factors in SDF. These limitations can be managed in future prospective studies to elucidate the effects of other contributing factors in pathogenesis of SDF in Saudi population.

\section{CONCLUSION}

This was the first Saudi multicenter study considering the WHO 2009 dengue classification in the assessment of risk factors of SDF and showed that dengue is a significant health problem for Saudi population in Jeddah as well as for international health as Jeddah is a Haj access point with many pilgrims coming from high-burden dengue countries and from other worldwide dengue-endemic areas. Predominant infection of adults and males with dengue was detected in this study. There were few SDF cases with low CFR mostly because of well-established and effective Saudi surveillance. Consideration of warning signs for severe dengue may result in hospital admission, prompting closer monitoring, timely and proper interventions and reduced mortality in SDF cases. Pediatric cases had significantly more risk to develop SDF, but degree of severity of illness seemed to be slightly higher in adults. Multivariate regression analysis detected that the most significant predictors of SDF in adults compared with children were significantly more SDI, significantly less clinical fluid accumulation, and significantly less neutropenia. The predictability for SDF of this logistic model reached $94.1 \%$ in adults with overall accuracy of $77.4 \%$. Moreover, in the absence of currently readily available vaccines of highly proven efficacy, specific therapies, and accurate point-of-care diagnostic tools for dengue all over the world, simple inexpensive strategies depending on proper assessment of dengue patients on initial presentation by warning signs of the WHO 2009 dengue classification and routine laboratory tests may assist clinicians in triage of patients to determine admission of cases at risk of SDF development particularly in resource-poor settings or during extensive outbreaks and provide these cases with early continuous monitoring and intensive care to save the lives of SDF cases.

TABLE 4

Multivariate logistic regression analysis for risk factors or predictors of severe dengue in adults vs. children

\begin{tabular}{|c|c|c|c|c|c|c|}
\hline \multirow[b]{2}{*}{ Predictor } & \multirow[b]{2}{*}{ Estimate } & \multirow[b]{2}{*}{$z$} & \multirow[b]{2}{*}{$P$-value } & \multirow[b]{2}{*}{ Adjusted odds ratio } & \multicolumn{2}{|c|}{$95 \% \mathrm{Cl}$} \\
\hline & & & & & Lower confidence limit & Upper confidence limit \\
\hline Intercept & 1.71 & 1.87 & 0.06 & 5.54 & 0.92 & 33.43 \\
\hline Male gender & 0.34 & 0.85 & 0.39 & 1.40 & 0.64 & 3.05 \\
\hline Secondary dengue infection & 0.79 & 2.19 & 0.02 & 2.20 & 1.09 & 4.44 \\
\hline Persistent vomiting & -0.72 & -0.71 & 0.48 & 0.49 & 0.07 & 3.53 \\
\hline Shock & 0.15 & 0.20 & 0.84 & 1.17 & 0.26 & 5.16 \\
\hline Bleeding & 0.09 & 0.17 & 0.86 & 1.10 & 0.39 & 3.11 \\
\hline Clinical fluid accumulation & -1.75 & -3.69 & $<0.001$ & 0.17 & 0.07 & 0.44 \\
\hline Organ failure & 0.21 & 0.50 & 0.62 & 1.23 & 0.54 & 2.81 \\
\hline White blood cell count & 0.08 & 0.83 & 0.40 & 1.08 & 0.90 & 1.29 \\
\hline Neutropenia & -0.90 & -2.02 & 0.04 & 0.41 & 0.17 & 0.97 \\
\hline
\end{tabular}




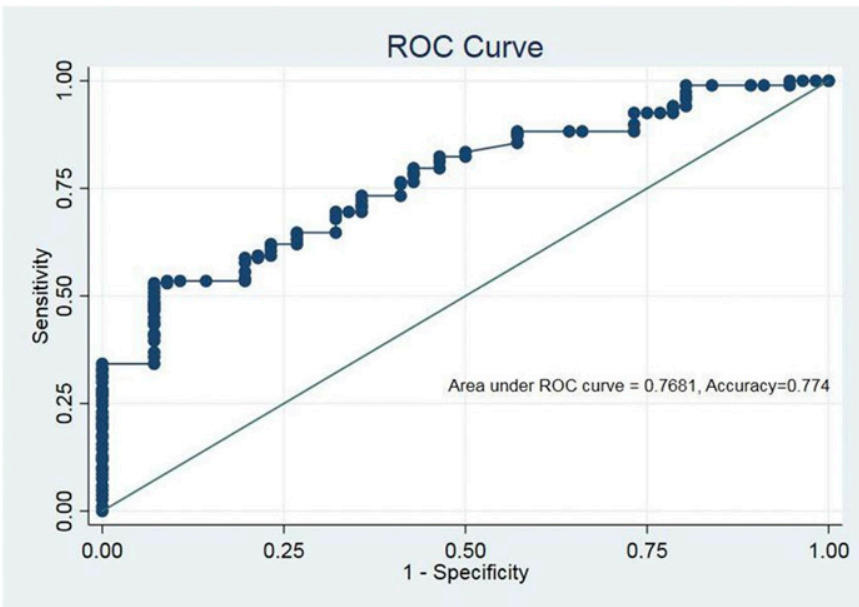

FIGURE 3. Receiver operating characteristic $(\mathrm{ROC})$ curve showing predictive accuracy of multivariate logistic regression model. The predictability for SDF of this logistic model reached $94.1 \%$ in adults with an overall accuracy of $77.4 \%$. Hosmer-Lemeshow test was applied to check the fitness of the multiple logistic regression model $\left(X^{2}=12.54\right.$, degree of freedom $\left.=8, P=0.129\right)$, indicating that the model was fit. Area under ROC curve was 0.768 , implying acceptable discrimination of the model. This figure appears in color at www.ajtmh.org.

Received September 1, 2019. Accepted for publication November 29, 2019.

Published online January 13, 2020.

Acknowledgments: This project was funded by the Deanship of Scientific Research (DSR) at King Abdulaziz University, Jeddah, under grant no. (G-559- 828-1439). The authors, therefore, acknowledge with thanks DSR for technical and financial support

Disclaimer: DSR did not have any role in the study design; in the collection, analysis, or interpretation of data; in the writing of the article.

Authors' addresses: Moustafa A. Hegazi, Department of Pediatrics, Faculty of Medicine in Rabigh, King Abdulaziz University, Jeddah, Saudi Arabia, and Department of Pediatrics, Mansoura University Children's Hospital, Mansoura, Egypt, E-mail: mhegazi712003@ yahoo.co.uk. Marwan A. Bakarman and Nadeem S. Butt, Department of Family and Community Medicine, Faculty of Medicine in Rabigh, King Abdulaziz University, Jeddah, Saudi Arabia, E-mails: mbakarman@kau.edu.sa and nshafique@kau.edu.sa. Turki S. Alahmadi, Department of Pediatrics, Faculty of Medicine in Rabigh, King Abdulaziz University, Jeddah, Saudi Arabia, and Department of Pediatrics, King Abdulaziz University Hospital, Jeddah, Saudi Arabia, E-mail: tsalahmadi@kau.edu.sa. Ahmed M. Alqahtani, Badr S. Aljedaani, and Abdulrahman H. Almajnuni, Faculty of Medicine in Rabigh, King Abdulaziz University, Saudi Arabia, E-mails: ahmed19941125@gmail.com, badr.com5@gmail.com, and drx2j4@gmail.com.

\section{REFERENCES}

1. World Health Organization, 2009. Dengue: Guidelines for Diagnosis, Treatment, Prevention and Control. Available at: https://www. who.int/tdr/publications/documents/dengue-diagnosis.pdf. Accessed January 15, 2017.

2. Pozo-Aguilar JO, Monroy-Martínez V, Díaz D, Barrios-Palacios J, Ramos C, Ulloa-García A, García-Pillado J, Ruiz-Ordaz BH, 2014. Evaluation of host and viral factors associated with severe dengue based on the 2009 WHO classification. Parasit Vectors 7: 590.

3. Yacoub S, Wills $B, 2014$. Predicting outcome from dengue. BMC Med 12: 147.

4. Narvaez F, Gutierrez G, Pérez MA, Elizondo D, Nuñez A, Balmaseda A, Harris E, 2011. Evaluation of the traditional and revised WHO classifications of dengue disease severity. PLOS Negl Trop Dis 5: e1397.

5. Guzman MG, Alvarez M, Halstead SB, 2013. Secondary infection as a risk factor for dengue hemorrhagic fever/dengue shock syndrome: an historical perspective and role of antibodydependent enhancement of infection. Arch Virol 158: 14451459.

6. Hammond SN et al., 2005. Differences in dengue severity in infants, children, and adults in a 3-year hospital-based study in Nicaragua. Am J Trop Med Hyg 73: 1063-1070.

7. Huy NT, Van Giang T, Thuy DH, Kikuchi M, Hien TT, Zamora J, Hirayama K, 2013. Factors associated with dengue shock syndrome: a systematic review and meta-analysis. PLoS Negl Trop Dis 7: e2412.

8. Fakeeh M, Zaki AM, 2001. Virologic and serologic surveillance for dengue fever in Jeddah, Saudi Arabia, 1994-1999. Am J Trop Med Hyg 65: 764-767.

9. Alzahrani AG, Al Mazroa MA, Alrabeah AM, Ibrahim AM, Mokdad $\mathrm{AH}$, Memish ZA, 2013. Geographical distribution and spatiotemporal patterns of dengue cases in Jeddah Governorate from 2006-2008. Trans $R$ Soc Trop Med Hyg 107: 23-29.

10. Ahmed MM, 2010. Clinical profile of dengue fever infection in King Abdul Aziz University Hospital Saudi Arabia. J Infect Dev Ctries 4: 503-510.

11. Vaughn DW, Nisalak A, Solomon T, Kalayanarooj S, Nguyen MD, Kneen R, Cuzzubbo A, Devine PL, 1999. Rapid serologic diagnosis of dengue virus infection using a commercial capture ELISA that distinguishes primary and secondary infections. Am J Trop Med Hyg 60: 693-698.

12. Tee HP, How SH, Jamalludin AR, Safhan MN, Sapian MM, Kuan YC, Sapari S, 2009. Risk factors associated with development of dengue haemorrhagic fever or dengue shock syndrome in adults in Hospital Tengku Ampuan Afzan Kuantan. Med $J$ Malaysia 64: 316-320.

13. Nguyen THT et al., 2018. Methods to discriminate primary from secondary dengue during acute symptomatic infection. BMC Infect Dis 18: 375.

14. Wichmann $\mathrm{O}$, Hongsiriwon S, Bowonwatanuwong $\mathrm{C}$, Chotivanich K, Sukthana Y, Pukrittayakamee S, 2004. Risk factors and clinical features associated with severe dengue infection in adults and children during the 2001 epidemic in Chonburi, Thailand. Trop Med Int Health 9: 1022-1029.

15. Singla N, Chaudhary P, Thakur M, Chander J, 2016. Dengue: an analysis of epidemiological pattern over a six year period. $J$ Clin Diagn Res 10: DC12-DC14.

16. Badreddine S, Al-Dhaheri F, Al-Dabbagh A, Al-Amoudi A, AlAmmari $M$, Elatassi $N$, Abbas $H$, Magliah R, Malibari $A$, Almoallim H, 2017. Dengue fever. Clinical features of 567 consecutive patients admitted to a tertiary care center in Saudi Arabia. Saudi Med J 38: 1025-1033.

17. Hashem AM, Abujamel T, Alhabbab R, Almazroui M, Azhar El, 2018. Dengue infection in patients with febrile illness and its 
relationship to climate factors: a case study in the city of Jeddah, Saudi Arabia, for the period 2010-2014. Acta Trop 181: 105-111.

18. Wills BA et al., 2005. Comparison of three fluid solutions for resuscitation in dengue shock syndrome. $N$ Engl $J$ Med 353: 877-889.

19. Kumar A, Gittens-St Hilaire M, Clarke-Greenidge N, Nielsen AL, 2015. Epidemiological trend and clinical observations among children and adults with dengue in Barbados. West Indian Med J 64: 37-42.

20. Mallhi TH, Khan AH, Sarriff A, Adnan AS, Khan YH, 2017. Determinants of mortality and prolonged hospital stay among dengue patients attending tertiary care hospital: a cross sectional retrospective analysis. BMJ Open 7: e016805.

21. Souza LJ, Pessanha LB, Mansur LC, Souza LA, Ribeiro MB, Silveira Mdo V, Souto Filho JT, 2013. Comparison of clinical and laboratory characteristics between children and adults with dengue. Braz J Infect Dis 17: 27-31.

22. Dejnirattisai $W$ et al., 2010. Cross reacting antibodies enhance dengue virus infection in humans. Science 328: 745-748.

23. Thein TL, Leo YS, Fisher DA, Low JG, Oh HM, Gan VC, Wong JG, Lye DC, 2013. Risk factors for fatality among confirmed adult dengue inpatients in Singapore: a matched case-control study. PLoS One 8: e81060.
24. Pinto RC, Castro DB, Albuquerque BC, Sampaio Vde S, Passos RA, Costa CF, Sadahiro M, Braga JU, 2016. Mortality predictors in patients with severe dengue in the State of Amazonas, Brazil. PLoS One 11: e0161884.

25. Rojas EM et al., 2019. Clinical indicators of fatal dengue in two endemic areas of Colombia: a hospital-based case-control study. Am J Trop Med Hyg 100: 411-419.

26. Lee IK, Liu JW, Yang KD, 2012. Fatal dengue hemorrhagic fever in adults: emphasizing the evolutionary pre-fatal clinical and laboratory manifestations. PLoS Negl Trop Dis 6: e1532.

27. Jaenisch $\mathrm{T}$ et al., 2016. Clinical evaluation of dengue and identification of risk factors for severe disease: protocol for a multicentre study in 8 countries. BMC Infect Dis 16: 120.

28. Sahana KS, Sujatha $R, 2012$. Clinical profile of dengue among children according to revised WHO classification: analysis of a 2012 outbreak from Southern India. Indian J Pediatr 82: 109-113.

29. Sreenivasan P, S G, K S, 2018. Development of a prognostic prediction model to determine severe dengue in children. Indian $J$ Pediatr 85: 433-439.

30. Trung DT et al., 2012. Clinical features of dengue in a large Vietnamese cohort: intrinsically lower platelet counts and greater risk for bleeding in adults than children. PLoS Negl Trop Dis 6: e1679. 